To be published in Ann. of NYAS, Fundamental Problems in Quantum Theory, D. Greenberger, (ed.) 1994; hep-th/9411196

\title{
PROTECTIVE MEASUREMENTS
}

\author{
Yakir Aharonov ${ }^{a, b}$ and Lev Vaidman ${ }^{a}$ \\ ${ }^{a}$ School of Physics and Astronomy \\ Raymond and Beverly Sackler Faculty of Exact Sciences \\ Tel-Aviv University, Tel-Aviv, 69978 ISRAEL \\ ${ }^{b}$ Physics Department, University of South Carolina \\ Columbia, South Carolina 29208, U.S.A.
}

\begin{abstract}
Protective measurements yield properties of the quantum state of a single quantum system without affecting the quantum state. A protective measurement involves adiabatic coupling to the measuring device together with a procedure to protect the state from changing. For nondegenerate energy eigenstates the protection is provided by the system itself. In this case it is actually possible to measure the Schrödinger wave via measurements on a single system. This fact provides an argument in favor of associating physical reality with a quantum state of a single system, challenging the usual ensemble interpretation. We also believe that the complete description of a quantum system requires a two-state vector formalism involving (in addition to the usual one) a future quantum state evolv-
\end{abstract}


ing backwards in time. Protective measurements testing the two-state vector reality are constructed. 


\section{INTRODUCTION}

Recently, we have proposed protective measurements ${ }^{1,2}$ that allow measuring the Schrödinger wave of a single particle. We have argued that the possibility of such measurements tells us that a quantum state has more physical meaning than is usually assumed; that is, the Schrödinger wave is real in some sense. A quantum state is not only a statistical property of an ensemble, it is a property of a single system.

Also, in recent years we developed an approach in which a quantum system is described, at a given time, by two (instead of one) quantum states: the usual one evolving toward the future and the second evolving backwards in time from a future measurement. ${ }^{3-8}$ This approach proved itself fruitful at least for describing measurements performed on preand post-selected ensembles. In this approach, the vector describing a quantum system at a given time consists of two states.

The following questions arise: Is there a contradiction between these two approaches? Which description is appropriate: the standard, single-state, or our two-state description? Does the two-state vector have physical meaning for a single system? Is it possible to measure this vector on a single system? In this work we shall try to answer these questions.

In the following section we present our method of protective measurements of a single quantum state. This is followed by a brief review of the two-state vector formalism. Then, the main result of this work - the method of protective measurement of a two-state vector - is presented. We conclude with a discussion of the obtained results. 


\section{MEASUREMENT OF THE SCHRÖDINGER WAVE OF A SINGLE PARTICLE}

At present, the commonly accepted interpretation of the Schrödinger wave is due to Born. He proposed to interpret the wave intensity not as the density of distribution of actual matter, as Schrödinger first imagined, but as a probability density for the presence of a particle. Schrödinger, however, wanted to believe that his wave represents a single particle: the wave is an extended object really moving in space. Born's interpretation was supported by the fact that nobody knew how to measure the density of the Schrödinger wave on a single system. There was a general belief that the Schrödinger wave could only be tested for an ensemble of particles. We have proposed a new type of measurements: "protective measurements" that allow direct measurement of the Schrödinger wave density on a single particle. We have shown that one can simultaneously measure the density and the current of the Schrödinger wave in many locations. The results of these measurements then allow the reconstruction of the Schrödinger wave.

The simplest protection procedure is introducing a protective potential such that the quantum state of the system will be a nondegenerate eigenstate of the Hamiltonian. In fact, in many important cases this protection is given by nature: almost isolated systems will eventually decay to their ground state or to some stable excited state.

As an example of a simple protective measurement, let us consider a particle in a discrete nondegenerate energy eigenstate $\Psi(x)$. The standard von Neumann procedure for measuring the value of an observable $A$ in this state involves an interaction Hamiltonian,

$$
H=g(t) P A
$$

coupling the system to a measuring device, or pointer, with coordinate and momentum denoted, respectively, by $Q$ and $P$. The time-dependent coupling $g(t)$ is normalized to $\int g(t) d t=1$, and the initial state of the pointer is taken to be a Gaussian centered around 
zero.

In standard impulsive measurements, $g(t) \neq 0$ for only a very short time interval. Thus, the interaction term dominates the rest of the Hamiltonian, and the time evolution $e^{-\frac{i}{\hbar} P A}$ leads to a correlated state: eigenstates of $A$ with eigenvalues $a_{n}$ are correlated to measuring device states in which the pointer is shifted by these values $a_{n}$. By contrast, the protective measurements of interest here utilize the opposite limit of extremely slow measurement. We take $g(t)=1 / T$ for most of the time $T$ and assume that $g(t)$ goes to zero gradually before and after the period $T$. We choose the initial state of the measuring device such that the canonical conjugate $P$ (of the pointer variable $Q$ ) is bounded. We also assume that $P$ is a constant of motion not only of the interaction Hamiltonian (1), but of the whole Hamiltonian. For $g(t)$ smooth enough we obtain an adiabatic process in which the particle cannot make a transition from one energy eigenstate to another, and, in the limit $T \rightarrow \infty$, the interaction Hamiltonian does not change the energy eigenstate. For any given value of $P$, the energy of the eigenstate shifts by an infinitesimal amount given by first order perturbation theory:

$$
\delta E=\left\langle H_{\text {int }}\right\rangle=\frac{\langle A\rangle P}{T} .
$$

The corresponding time evolution $e^{-i P\langle A\rangle / \hbar}$ shifts the pointer by the average value $\langle A\rangle$. This result contrasts with the usual (strong) measurement in which the pointer shifts by one of the eigenvalues of $A$. By measuring the averages of a sufficiently large number of variables $A_{n}$, the full Schrödinger wave $\Psi(x)$ can be reconstructed to any desired precision.

As a specific example we take the $A_{n}$ to be (normalized) projection operators on small regions $V_{n}$ having volume $v_{n}$ :

$$
A_{n}= \begin{cases}\frac{1}{v_{n}}, & \text { if } x \in V_{n}, \\ 0, & \text { if } x \notin V_{n} .\end{cases}
$$


The measurement of $A_{n}$ yields

$$
\left\langle A_{n}\right\rangle=\frac{1}{v_{n}} \int_{V_{n}}|\Psi|^{2} d v=\left|\Psi_{n}\right|^{2}
$$

where $\left|\Psi_{n}\right|^{2}$ is the average of the density $\rho(x)=|\Psi(x)|^{2}$ over the small region $V_{n}$. Performing measurements in sufficiently many regions $V_{n}$ we can reconstruct $\rho(x)$ everywhere in space. (Simultaneous measurement of all the variables $A_{n}$ requires slower and weaker interactions, and thus takes more time.) For a real state the density $\rho(x)$ is itself enough to reconstruct the Schrödinger wave; we can fix the sign by flipping it across nodal surfaces.

In the general case, however, we have to measure current density in addition to measurements of the density $\rho(x)$. This time we also adiabatically measure the averages of

$$
B_{n}=\frac{1}{2 i}\left(A_{n} \nabla+\nabla A_{n}\right)
$$

Indeed, $\left\langle B_{n}\right\rangle$ are the average values of the current $j=\frac{1}{2 i}\left(\Psi^{*} \nabla \Psi-\Psi \nabla \Psi^{*}\right)$ in the region $V_{n}$. Writing $\Psi(x)=r(x) e^{i \theta(x)}$ with $r(x)=\sqrt{\rho(x)}$, we find that

$$
\frac{j(x)}{\rho(x)}=\nabla \theta \quad ;
$$

and the phase $\theta(x)$ can be found by integrating $j / \rho$.

For a charged particle, the density $\rho(x)$ times the charge yields the effective charge density. In particular, it means that an appropriate adiabatic measurement of the Gauss flux out of a certain region must yield the expectation value of the charge inside this region (the integral of the charge density over this region). Likewise, adiabatic measurement of the Ampere contour integral yields the expectation value of the total current flowing through this contour in the stationary case.

Our procedure is not applicable to degenerate energy eigenstates. The simplest way to deal with this case is by adding a potential (as part of the measuring procedure) to lift 
the degeneracy. This protection does not change the state. However, one can argue that it changes the physical situation. We can bring this change to a minimum by adding strong protection potential for a dense set of very short time intervals. Thus, most of the time the system has not only the same state, but also the original potential.

We can measure even a superposition of energy eigenstates by a similar procedure. We add a dense set of time-dependent potentials acting for very short periods of time such that the state at all these times is the eigenstate of the Hamiltonian together with the additional potential. Still, most of the time the system evolves under the free Hamiltonian. The proof of the efficiency of the above strong impulsive potentials is similar to the proof of the Zeno "paradox" in which a quantum system under a dense set of observations evolves in accordance with the evolution tested, and not according to the free Hamiltonian. In our case, the two evolutions are identical.

\section{TWO-STATE VECTOR DESCRIPTION OF A QUANTUM SYSTEM}

In 1964 Aharonov, Bergmann and Lebowitz ${ }^{9}$ considered measurements performed on a quantum system between two other measurements, results of which were given. They proposed describing the quantum system between two measurements by using two states: the usual one, evolving towards the future from the time of the first measurement, and a second state evolving backwards in time, from the time of the second measurement. If a system has been prepared at time $t_{1}$ in a state $\left|\Psi_{1}\right\rangle$ and is found at time $t_{2}$ in a state $\left|\Psi_{2}\right\rangle$, then at time $t, t_{1}<t<t_{2}$, the system is described by

$$
\left\langle\Psi_{2}\right| e^{i \int_{t_{2}}^{t} H d t} \text { and } e^{-i \int_{t_{1}}^{t} H d t}\left|\Psi_{1}\right\rangle \text {. }
$$

For simplicity, we shall consider the free Hamiltonian to be zero; then, the system at time $t$ is described by the two states $\left\langle\Psi_{2}\right|$ and $\left|\Psi_{1}\right\rangle$. In order to obtain such a system, we 
prepare an ensemble of systems in the state $\left|\Psi_{1}\right\rangle$, perform a measurement of the desired variable using separate measuring devices for each system in the ensemble, and perform the post-selection measurement. If the outcome of the post-selection was not the desired result, we discard the system and the corresponding measuring device. We look only at measuring devices corresponding to the systems post-selected in the state $\left\langle\Psi_{2}\right|$.

The basic concepts of the two-state approach, the weak value of a physical variable $A$ in the time interval between pre-selection of the state $\left|\Psi_{1}\right\rangle$ and post-selection of the state $\left|\Psi_{2}\right\rangle$ is given by

$$
A_{w} \equiv \frac{\left\langle\Psi_{2}|A| \Psi_{1}\right\rangle}{\left\langle\Psi_{2} \mid \Psi_{1}\right\rangle}
$$

Let us present the main idea by way of a simple example. We consider, at time $t$, a quantum system that was prepared at time $t_{1}$ in the state $|B=b\rangle$ and that was found at time $t_{2}$ in the state $|C=c\rangle, t_{1}<t<t_{2}$. The measurements at times $t_{1}$ and $t_{2}$ are complete measurements of, in general, noncommuting variables $B$ and $C$. The free Hamiltonian is zero, and therefore, the first quantum state at time $t$ is $|B=b\rangle$. In the two-state approach we characterize the system at time $t$ by backwards-evolving state $\langle C=c|$ as well. Our motivation for including the future state is as follws: if we know that a measurement of $C$ has been performed at time $t$ then the outcome is $C=c$ with probability 1 . This intermediate measurement, however, destroys our knowledge that $B=b$, since the coupling of the measuring device to the variable $C$ can change $B$. The idea of weak measurements is to make the coupling with the measuring device sufficiently weak so that $B$ does not change. In fact, we require that both quantum states do not change, neither the usual one $|B=b\rangle$ evolving towards the future nor $\langle C=c|$ evolving backwards.

During the whole time interval between $t_{1}$ and $t_{2}$, both $B=b$ and $C=c$ are true (in some sense). But then, $B+C=b+c$ must also be true. The latter statement, 
however, might not have meaning in the standard quantum formalism because the sum of the eigenvalues $b+c$ might not be an eigenvalue of the operator $B+C$. An attempt to measure $B+C$ using a standard measuring procedure will lead to some change of the two quantum states and thus the outcome will not be $b+c$. A weak measurement, however, will yield $b+c$.

When the "strong" value of an observable is known with certainty, that is, we know the outcome of an ideal (infinitely strong) measurement with probability 1 , the weak value is equal to the strong value. Let us analyze the example above. The strong value of $B$ is $b$, its eigenvalue. The strong value of $C$ is $c$, as we know from retrodiction. From the definition (1) immediately follows: $B_{w}=b$ and $C_{w}=c$. However, weak values, unlike strong values, are defined not just for $B$ and $C$, but for all operators. The strong value of the sum $B+C$ when $[B, C] \neq 0$ is not defined, but the weak value of the sum is defined: $(B+C)_{w}=b+c$.

The system at time $t$ in a pre- and post-selected ensemble is defined by two states, the usual one evolving from the time of the preparation and the state evolving backwards in time from the post-selection. We may neglect the free Hamiltonian if the time between the pre-selection and the post-selection is very short. Consider a system that has been pre-selected in a state $\left|\Psi_{1}\right\rangle$ and shortly afterwards post-selected in a state $\left|\Psi_{2}\right\rangle$. The weak value of any physical variable $A$ in the time interval between the pre-selection and the post-selection is given by Eq. (8). Let us show briefly how weak values emerge from a measuring procedure with a sufficiently weak interaction.

We consider a sequence of measurements: a pre-selection of $\left|\Psi_{1}\right\rangle$, a (weak) measurement interaction of the form of Eq. (1), and a post-selection measurement finding the state $\left|\Psi_{2}\right\rangle$. The state of the measuring device (which was initially in a Gaussian state) after this 
sequence is given (up to normalization) by

$$
\Phi(Q)=\left\langle\Psi_{2}\left|e^{-i P A}\right| \Psi_{1}\right\rangle e^{-Q^{2} / 2 \Delta^{2}}
$$

After simple algebraic manipulation we can rewrite it (in the $P$-representation) as

$$
\tilde{\Phi}(P)=\left\langle\Psi_{2} \mid \Psi_{1}\right\rangle e^{-i A_{w} P} e^{-\Delta^{2} P^{2} / 2}+\left\langle\Psi_{2} \mid \Psi_{1}\right\rangle \sum_{n=2}^{\infty} \frac{(i P)^{n}}{n !}\left[\left(A^{n}\right)_{w}-\left(A_{w}\right)^{n}\right] e^{-\Delta^{2} P^{2} / 2}
$$

If $\Delta$ is sufficiently large, then we can neglect the second term of (10) when we Fourier transform back to the $Q$-representation. Large $\Delta$ corresponds to weak measurement in the sense that the interaction Hamiltonian (1) is small. Thus, in the limit of weak measurement, the final state of the measuring device (in the $Q$-representation) is

$$
\Phi(Q)=\left(\Delta^{2} \pi\right)^{-1 / 4} e^{-\left(Q-A_{w}\right)^{2} / 2 \Delta^{2}}
$$

This state represents a measuring device pointing to the weak value, $A_{w}$.

Although we have showed this result for a specific von Neumann model of measurements, the result is completely general: any coupling of a pre- and post-selected system to a variable $A$, provided the coupling is sufficiently weak, results in effective coupling to $A_{w}$. This weak coupling between a single system and the measuring device will not, in most cases, lead to a distinguishable shift of the pointer variable, but collecting the results of measurements on an ensemble of pre- and post-selected systems will yield the weak values of a measured variable to any desired precision.

When the strength of the coupling to the measuring device goes to zero, the outcomes of the measurement invariably yield the weak value. To be more precise, a measurement yields the real part of the weak value. Indeed, the weak value is, in general, a complex number, but its imaginary part will contribute only a phase to the wave function of the measuring device in the position representation of the pointer. Therefore, the imaginary 
part will not affect the probability distribution of the pointer position, which is what we see in a usual measurement. However, the imaginary part of the weak value also has physical meaning. It expresses itself as a change in the conjugate momentum of the pointer variable. ${ }^{7}$

Let us consider a measurement of a spin component of a spin- $1 / 2$ particle. We shall consider a particle prepared in the initial state spin "up" in the $\hat{x}$ direction and postselected to be "up" in the $\hat{y}$ direction. At the intermediate time we measure, weakly, the spin component in the $\hat{\xi}$ direction which is bisector of $\hat{x}$ and $\hat{y}$, that is, $\sigma_{\xi}=\left(\sigma_{x}+\sigma_{y}\right) / \sqrt{2}$. Thus $\left|\Psi_{1}\right\rangle=\left|\uparrow_{x}\right\rangle,\left|\Psi_{2}\right\rangle=\left|\uparrow_{y}\right\rangle$, and the weak value of $\sigma_{\xi}$ in this case is:

$$
\left(\sigma_{\xi}\right)_{w}=\frac{\left\langle\uparrow_{y}\left|\sigma_{\xi}\right| \uparrow_{x}\right\rangle}{\left\langle\uparrow_{y} \mid \uparrow_{x}\right\rangle}=\frac{1}{\sqrt{2}} \frac{\left\langle\uparrow_{y}\left|\left(\sigma_{x}+\sigma_{y}\right)\right| \uparrow_{x}\right\rangle}{\left\langle\uparrow_{y} \mid \uparrow_{x}\right\rangle}=\sqrt{2} .
$$

This value is, of course, "forbidden" in the standard interpretation where a spin component can obtain the (eigen) values \pm 1 only.

The Hamiltonian for measuring $\sigma_{\xi}$ is

$$
H=g(t) P \sigma_{\xi}
$$

After the measuring interaction, the quantum state of the system and the pointer of the measuring device is

$$
\cos (\pi / 8)\left|\uparrow_{\xi}\right\rangle e^{-(Q-1)^{2} / 2 \Delta^{2}}+\sin (\pi / 8)\left|\downarrow_{\xi}\right\rangle e^{-(Q+1)^{2} / 2 \Delta^{2}}
$$

The probability distribution of the pointer position, if it is observed now without postselection, is the sum of the distributions for each spin value. It is, up to normalization,

$$
\operatorname{prob}(Q)=\cos ^{2}(\pi / 8) e^{-(Q-1)^{2} / \Delta^{2}}+\sin ^{2}(\pi / 8) e^{-(Q+1)^{2} / \Delta^{2}} .
$$

In the usual strong measurement, $\Delta \ll 1$. In this case, the probability distribution of the pointer is localized around -1 and +1 and it is strongly correlated to the values of the spin, $\sigma_{z}= \pm 1$. 
Weak measurement corresponds to a $\Delta$ that is much larger than the range of the eigenvalues, that is, $\Delta \gg 1$. The pointer distribution has a large uncertainty, and it is peaked between the eigenvalues, more precisely, at the expectation value $\left\langle\uparrow_{x}\left|\sigma_{\xi}\right| \uparrow_{x}\right\rangle=$ $1 / \sqrt{2}$. An outcome of an individual measurement usually will not be close to this number, but it can be found from an ensemble of such measurements. Note, that we have not yet considered the post-selection.

In order to simplify the analysis of measurements on the pre- and post-selected ensemble, let us assume that we first make the post-selection of the spin of the particle and only then look at the pointer of the device that weakly measures $\sigma_{\xi}$. We must get the same result as if we first look at the outcome of the weak measurement, make the postselection, and discard all readings of the weak measurement corresponding to the cases in which the result is not $\sigma_{y}=1$. The post-selected state of the particle in the $\sigma_{\xi}$ representation is $\left|\uparrow_{y}\right\rangle=\cos (\pi / 8)\left|\uparrow_{\xi}\right\rangle-\sin (\pi / 8)\left|\downarrow_{\xi}\right\rangle$. The state of the measuring device after the post-selection of the spin state is obtained by projection of (14) onto the post-selected state:

$$
\Phi(Q)=\mathcal{N}\left(\cos ^{2}(\pi / 8) e^{-(Q-1)^{2} / 2 \Delta^{2}}-\sin ^{2}(\pi / 8) e^{-(Q+1)^{2} / 2 \Delta^{2}}\right)
$$

where $\mathcal{N}$ is a normalization factor. The probability distribution of the pointer variable is given by

$$
\operatorname{prob}(Q)=\mathcal{N}^{2}\left(\cos ^{2}(\pi / 8) e^{-(Q-1)^{2} / 2 \Delta^{2}}-\sin ^{2}(\pi / 8) e^{-(Q+1)^{2} / 2 \Delta^{2}}\right)^{2} .
$$

If the measuring interaction is strong, that is, $\Delta \ll 1$, then the distribution is localized around the eigenvalues \pm 1 (mostly around 1 because the pre- and post-selected probability to find $\sigma_{\xi}=1$ is more than $85 \%$ ), see Figs. $1 a, 1 b$. But when the strength of the coupling is weakened, that is, $\Delta$ is increased, the distribution gradually changes to a single broad peak around $\sqrt{2}$, the weak value, see Figs. $1 c-1 e$. 
The width of the peak is large and therefore each individual reading of the pointer usually will be pretty far from $\sqrt{2}$. The physical meaning of the weak value, in this case, can be associated only with an ensemble of pre- and post-selected particles. The accuracy of defining the center of the distribution goes as $1 / \sqrt{N}$; thus, bu increasing $N$, the number of particles in the ensemble, we can find the weak value with any desired precision, see Fig. $1 f$.

\section{PROTECTION OF A TWO-STATE VECTOR}

We are familiar with weak measurements performed on a single system. In fact, the first work on weak measurements ${ }^{3}$ considered such a case. We have shown how a single measurement of the spin component of a spin- $N$ system could yield the "forbidden" value $\sqrt{2} N$ with the uncertainty $\sqrt{N}$. This is the weak value of $S_{\xi}$ for the two-state vector $\left\langle S_{y}=N \| S_{x}=N\right\rangle$. Another example that we have investigated is the measurement of the kinetic energy of a tunneling particle. ${ }^{9}$ We have shown for any precision of the measurement that we can ensure a negative value reading of the measuring device by an appropriate choice of the post-selection state.

However, in these examples there is no measurement of two-state vector. If our measuring device for the spin measurement shows $\sqrt{2} N$, we cannot deduce that our two-state vector is $\left\langle S_{y}=N \| S_{x}=N\right\rangle$. Indeed, there are many other two-state vectors that yield the same weak value for the spin component, but we cannot even claim that we have one of these vectors, because the probability to obtain the "forbidden" outcome $S_{\xi}=\sqrt{2} N$ due to a statistical error of the measuring device is much higher. The same applies to the measurement of kinetic energy of a tunneling particle. The negative value shown by the measuring device usually is due to a statistical error, and only in very rare cases does it 
correspond to a particle "caught" in the tunneling process.

We could try to use several weak measurements on a single pre- and post-selected system in order to specify the two-state vector. But in that case these measurements will change the two-state vector. Therefore, as in the case of the measurement of the forward evolving single-state vector of a single system, we need a protection procedure.

At first look, it seems that protection of a two-state vector is impossible. Indeed, if we add a potential that makes one state to be a nondegenerate eigenstate, then the other state, if it is different, cannot be an eigenstate too (the states of the two-state vector cannot be orthogonal). The Zeno-type protection does not work either: if we test that the system is in one state then we know that it is not in another state. But, nevertheless, protection of the two-state vector is possible, as we will show next.

The procedure for protection of a two-state vector of a given system is carried out by coupling the system to another pre- and post-selected system. The protection procedure takes advantage of the fact that weak values might acquire complex values. Thus, the effective Hamiltonian of the protection might not be Hermitian. Non-Hermitian Hamiltonians act in different ways on quantum states evolving forward and backwards in time. This allows simultaneous protection of two different states (evolving in opposite time directions).

Let us start with the description of the protection of a two-state vector of a spin-1/2 particle considered previously, $\left\langle\uparrow_{y} \| \uparrow_{x}\right\rangle$. The protection procedure uses an external pre- and post-selected system $S$ of a large spin $N$ that is coupled to our spin via the interaction:

$$
H_{\text {prot }}=-\mathbf{S} \cdot \sigma
$$

The external system is pre-selected in the state $\left|S_{x}=N\right\rangle$ and post-selected in the state $\left\langle S_{y}=N\right|$, that is, it is described by the two-state vector $\left\langle S_{y}=N|| S_{x}=N\right\rangle$. When $N$ is large, 
and the interaction with our spin-1/2 particle is not too strong, the latter cannot change significantly the two-state vector of the protective system $S$, and the spin-1/2 particle "feels" the effective Hamiltonian in which $S$ is replaced by its weak value,

$$
\mathbf{S}_{w}=\frac{\left\langle S_{y}=N\left|\left(S_{x}, S_{y}, S_{z}\right)\right| S_{x}=N\right\rangle}{\left\langle S_{y}=N \mid S_{x}=N\right\rangle}=(N, N, i N)
$$

Thus, the effective protective Hamiltonian is:

$$
H_{e f f}=-N\left(\sigma_{x}+\sigma_{y}+i \sigma_{z}\right)
$$

Straightforward calculations show that this (non-Hermitian) Hamiltonian has two (nonorthogonal) eigenstates: $\left|\uparrow_{x}\right\rangle$ (with eigenvalue $-N$ ) and $\left|\downarrow_{y}\right\rangle$ (with eigenvalue $N$ ). This result provides a certain test of our approach. When we consider the original problem given by the Hamiltonian (18), we can easily see that if we start in the state $\left|\uparrow_{x}\right\rangle$ then all following measurements of $\sigma_{x}$ must yield value 1 , whereas if we start with the state $\left|\downarrow_{y}\right\rangle$ then all following measurements of $\sigma_{y}$ must yield the value -1 .

However, for backward evolving states the effective Hamiltonian is the hermitian conjugate of $(20)$ and it has different eigenstates: $\left\langle\uparrow_{y}\right|$ (with eigenvalue $-N$ ) and $\left\langle\downarrow_{x}\right|$ (with eigenvalue $N)$. Again, it is easily seen that if the particle is post-selected in the state $\left\langle\uparrow_{y}\right|$ then all preceding measurements of $\sigma_{y}$ must yield $\sigma_{y}=1$, whereas the post-selection of $\left|\downarrow_{x}\right\rangle$ ensure $\sigma_{x}=-1$ for all preceding measurements.

The two-state vectors $\left\langle\uparrow_{y} \| \uparrow_{x}\right\rangle$ and $\left\langle\downarrow_{x} \| \downarrow_{y}\right\rangle$ do not change under the action of the Hamiltonian (18). In order to prove that this Hamiltonian indeed provides the protection, we have to show that measuring interactions with the spin components of the particle will not lead to significant changes. For example, we must show that we can measure the weak value of $\sigma_{\xi}=\left(\sigma_{x}+\sigma_{y}\right) / \sqrt{2}$, which is $\left(\sigma_{\xi}\right)_{w}=\sqrt{2}$, on a single particle. (As previously shown, without protection, the weak value is obtained only with an uncertainty that is 
larger than the observed value; therefore in order to find the weak value the pre- and post-selected ensemble has to be used, see Fig. 1.) The effective Hamiltonian during the measuring process is the sum of (1) and (20):

$$
H_{e f f}=-N\left(\sigma_{x}+\sigma_{y}+i \sigma_{z}\right)+\frac{P}{\sqrt{2}}\left(\sigma_{x}+\sigma_{y}\right)
$$

For any realistic measurement, $P$ is effectively bounded; thuus, for $N$ large enough, the second term will not change significantly the eigenvectors. The two-state vector $\left\langle\uparrow_{y} \| \uparrow_{x}\right\rangle$ will remain essentially unchanged during the measurement, and therefore the measuring device on this single particle will yield $\left(\sigma_{\xi}\right)_{w}=\sqrt{2}$. This weak value by itself is not enough to establish the two-state vector, but we can perform several weak measurements such as $\left(\sigma_{x}\right)_{w}=1,\left(\sigma_{y}\right)_{w}=1$, and $\left(\sigma_{z}\right)_{w}=i$ that uniquely define the two-state vector.

We have shown that the Hamiltonian (18), with an external system described by the two-state vector $\left\langle S_{y}=N \| S_{x}=N\right\rangle$, provides protection for the two-state vector $\left\langle\uparrow_{y} \| \uparrow_{x}\right\rangle$. It is not difficult to demonstrate that any two-state vector obtained by pre- and postselection of the spin-1/2 particle can be protected by the Hamiltonian (18). A general form of the two-state vector is $\left\langle\uparrow_{\beta} \| \uparrow_{\alpha}\right\rangle$ where $\hat{\alpha}$ and $\hat{\beta}$ denote some directions. It can be verified by a straightforward calculation that the two-state vector $\left\langle\uparrow_{\beta} \| \uparrow_{\alpha}\right\rangle$ is protected when the two-state vector of the protective device is $\left\langle S_{\beta}=N \| S_{\alpha}=N\right\rangle$.

One can naively suggest the following simple explanation of the above procedure. We pre-select the external system in a state $\left|S_{\alpha}=N\right\rangle$. Large $N$ corresponds to the classical limit, so this is equivalent to a "magnetic" field in the $-\hat{\alpha}$ direction. Thus, the quantum states of the system under study evolving to the future "feel" this strong magnetic field. The state of the system, $\left|\uparrow_{\alpha}\right\rangle$, is a ground state and therefore it is protected. Similarly, for the states evolving backwards in time, there is strong "magnetic" field in $-\hat{\beta}$ direction, protecting the state $\left\langle\uparrow_{\beta}\right|$. However, this picture is too naive. Based on this argument, one 
would expect that in addition to $\left|\uparrow_{\alpha}\right\rangle$ the forward evolving state $\left|\downarrow_{\alpha}\right\rangle$ is also protected, however, this is not so. There exists another forward evolving protected state, but it is $\left|\downarrow_{\beta}\right\rangle$. Also, in addition to $\left\langle\uparrow_{\beta}\right|$ there exists another protected backward evolving state, however, it is $\left\langle\downarrow_{\alpha}\right|$ and not the expected state $\left\langle\downarrow_{\beta}\right|$.

The failure of this naive explanation does not allow a simple protection scheme of the two-state vector of an arbitrary quantum system, $\left\langle\Psi_{2}|| \Psi_{1}\right\rangle$. According to this scheme, we construct a coupling of the system under study to the external system such that $\left|\Psi_{1}\right\rangle$ is a ground state when the external system is in a state $\left|\Phi_{1}\right\rangle$ and $\left\langle\Psi_{2}\right|$ is a ground (backward evolving) state when the external system is in a state $\left\langle\Phi_{2}\right|$. The difficulty here, namely, that the post-selection of the state $\left\langle\Psi_{2}\right|$ is impossible because usually in this situation $\left\langle\Phi_{2} \mid \Phi_{1}\right\rangle=0$, cannot be naively solved by adding a tiny component of the pre-selected state to the post-selected one, that is, post-selecting $\left\langle\Phi_{2}\right|+\epsilon\left\langle\Phi_{1}\right|$ instead of $\left\langle\Phi_{2}\right|$. Even for $\epsilon$ very small, the backward evolving state is not protected, and therefore the two-state vector is not protected either.

The proper way for protecting a two-state vector of an arbitrary system is a generalization of the protection procedure of the two-state vector of a spin- $1 / 2$ particle described above. The task is to protect a two-state vector $\left\langle\Psi_{2} \| \Psi_{1}\right\rangle$. Let us decompose the postselected state $\left|\Psi_{2}\right\rangle=a\left|\Psi_{1}\right\rangle+b\left|\Psi_{\perp}\right\rangle$. Now we can define "model spin" states: $\left|\Psi_{1}\right\rangle \equiv\left|\tilde{\uparrow}_{z}\right\rangle$ and $\left|\Psi_{\perp}\right\rangle \equiv\left|\tilde{\downarrow}_{z}\right\rangle$. On the basis of the two orthogonal states we can obtain all other "model spin" states. For example, $\left|\tilde{\uparrow}_{x}\right\rangle=1 / \sqrt{2}\left(\left|\tilde{\uparrow}_{z}\right\rangle+\left|\tilde{\downarrow}_{z}\right\rangle\right)$, and then we can define the "spin model" operator $\tilde{\sigma}$. Now, the protection Hamiltonian, in complete analogy with the spin- $1 / 2$ particle case is

$$
H_{\text {prot }}=-\mathbf{S} \cdot \tilde{\sigma}
$$

In order to protect the state $\left\langle\Psi_{2}|| \Psi_{1}\right\rangle$, the pre-selected state of the external system has to 
be $\left|S_{z}=N\right\rangle$ and the post-selected state has to be $\left\langle S_{\chi}=N\right|$ where the direction $\hat{\chi}$ is defined by the "spin model" representation of the state $\left|\Psi_{2}\right\rangle$ :

$$
\left|\tilde{\uparrow}_{\chi}\right\rangle \equiv\left|\Psi_{2}\right\rangle=\left\langle\Psi_{1} \mid \Psi_{2}\right\rangle\left|\tilde{\uparrow}_{z}\right\rangle+\left\langle\Psi_{\perp} \mid \Psi_{2}\right\rangle\left|\tilde{\downarrow}_{z}\right\rangle
$$

For general quantum states $\left|\Psi_{1}\right\rangle$ and $\left\langle\Psi_{2}\right|$, the required protection is a gedanken experiment. In general, the protection Hamiltonian (22) generates nonlocal interactions which can contradict relativistic causality. However, what we investigate here is a conceptual question in the framework of non-relativistic quantum theory, where any Hamiltonian is allowed.

\section{CONCLUSIONS}

We have shown in the framework of nonrelativistic quantum theory that we can measure (or, maybe a better word, "observe") two-state vectors describing pre- and postselected quantum systems. A number of (non-ideal) measurements define the two-state vector and we have a procedure to protect the two-state vector from significant change due to these measurements. In order to protect, we have to know the two-state vector. Thus, this procedure is also liable to the criticism ${ }^{11-12}$ leveled at our first proposal. Our response to this can be found in Ref. 13. Although we consider our present proposal as a measurement performed on a single system, it should also be mentioned that in any realistic practical implementation we will need ensembles of particles, protective systems, and measuring devices. The external system of the protective device has to be not only prepared (pre-selected) in a certain state, but also post-selected in a given state. In all interesting cases the probability for an appropriate outcome of the post-selection measurement is extremely small. Still, there is a non-zero probability that our first run with a single system, a single protective device, and a single set of measuring devices will yield 
the desired outcomes. In this case we have a reliable measurement performed on a single system. However, even when we use a pre-selected ensemble, we actually use only a single pre- and post-selected system. After achieving the first successful post-selection, we have completed the experiment. For more discussion of this point, see Ref. 14.

It is interesting to notice that our procedure cannot protect a generalized two-state vector $^{8}$ which is a superposition of two-state vectors. The system described by a generalized two-state vector is correlated to some external system. It seems that it is impossible to find any protective procedure of the generalized two-state vector that does not involve coupling to that external system. This feature hints that the generalized vector, although useful as a tool, is not a basic concept. The composite system consisting of the system under study and the system correlated to it is described by the usual, basic two-state vector.

Let us come back to the questions raised in the Introduction: is there a contradiction between "reality" of the Schrödinger wave, that is, the single-state vector, and "reality" of the two-state vector? Our answer is that the complete reality is described by the twostate vector. The single-state vector gives a partial description when we have only partial information. The apparent paradox of the descriptions is as follows. Consider a spin- $1 / 2$ particle described by the two-state vector $\left\langle\uparrow_{y}|| \uparrow_{x}\right\rangle$. The value $\sigma_{y}$ corresponding to this particle is $\sigma_{y}=1$. However, because it is described by the single (pre-selected) forward evolving state $\left|\uparrow_{x}\right\rangle$, the value of $\sigma_{y}$ is considered as the expectation value, $\left\langle\uparrow_{x}\left|\sigma_{y}\right| \uparrow_{x}\right\rangle=0$. According to our claims both are observable; thus, how can they be different?

In order to observe a quantum state it has to be protected. When we discussed the protective experiments of single-state vectors we did not say anything about quantum states evolving backwards in time. (It was not related to the point we wanted to make.) However, the protective procedure that we proposed, automatically protects identical back- 
ward evolving state. Thus, what we have proposed as an observation of a Schrödinger wave is indeed an observation a two-state vector with identical forward and backward evolving states. For example, the protection of spin-1/2 particle state, ${ }^{2}$ a strong magnetic field in a given direction, protects the two-state vector with either both states parallel or antiparallel to this direction. This procedure is incompatible with the protection of the forward evolving state parallel to one direction and the backward evolving state parallel to another. If the particle is described by $\left\langle\uparrow_{y} \| \uparrow_{x}\right\rangle$ then the strong magnetic field in the $\hat{x}$ direction will change of the backward evolving spin-state. There exists a protection procedure for $\left|\uparrow_{x}\right\rangle$ that does not change the backward evolving state as was described in the preceding section. The "observation" of the state protected in such a way will not yield the pre-selected quantum state but it will yield the picture defined by the two-state vector.

Thus, the contradiction is resolved by giving a more accurate interpretation of our original protective measurement of the Schrödinger wave. We observed not a single-state vector, but a two-state vector with identical backward and forward evolving states.

It is a pleasure to thank Sandu Popescu and Jacob Grunhaus for helpful discussions. This research was supported in part by grant 425/92-1 of the Basic Research Foundation (administered by the Israel Academy of Sciences and Humanities).

\section{REFERENCES}

1. Y. Aharonov and L. Vaidman, Phys. Lett. A178, 38 (1993).

2. Y. Aharonov, J. Anandan, and L. Vaidman, Phys. Rev. A 47, 4616 (1993).

3. Y. Aharonov, D. Albert, A. Casher, and L. Vaidman, Phys. Lett. A 124, 199 (1987).

4. L. Vaidman, Y. Aharonov and D. Albert Phys. Rev. Lett. 58, 1385 (1987).

5. Y. Aharonov, D. Albert, and L. Vaidman, Phys. Rev. Lett. 60, 1351 (1988). 
6. Y. Aharonov, J. Anandan, S. Popescu, and L. Vaidman, Phys. Rev. Lett. 64, 2965 (1990).

7. Y.Aharonov and L. Vaidman, Phys. Rev. A 41, 11 (1990).

8. Y.Aharonov and L. Vaidman, J. Phys. A 24, 2315 (1991).

9. Y. Aharonov, P.G. Bergmann and J.L. Lebowitz, Phys. Rev. B134, 1410 (1964).

10. Y. Aharonov, S. Popescu, D. Rohrlich, and L. Vaidman, Phys. Rev. A 48, 4084 (1993).

11. W. G. Unruh, Phys. Rev. A 50, 882 (1994).

12. C. Rovelli, Phys. Rev. A 50, 2788 (1994).

13. Y. Aharonov, J. Anandan, and L. Vaidman, The Meaning of the Protective Measurements, TAUP-2195-94, E-board hep-th/9408153 (1994).

14. L. Vaidman, in Advances in Quantum Phenomena, E. Beltrametti and J.M. LevyLeblond eds., NATO ASI series, Plenum, NY (1994). 


\section{FIGURE CAPTIONS}

Figure 1. Measurement on pre- and post-selected ensemble. Probability distribution of the pointer variable for measurement of $\sigma_{\xi}$ when the particle is pre-selected in the state $\left|\uparrow_{x}\right\rangle$ and post-selected in the state $\left|\uparrow_{y}\right\rangle$. The strength of the measurement is parameterized by the width of the distribution $\Delta$. (a) $\Delta=0.1 ;(b) \Delta=0.25 ;(c) \Delta=1 ;(d) \Delta=3$; (e) $\Delta=10 .(f)$ Weak measurement on the ensemble of 5000 particles; the original width of the peak, $\Delta=10$, is reduced to $10 / \sqrt{5} 000 \simeq 0.14$. In the strong measurements $(a)-(b)$ the pointer is localized around the eigenvalues \pm 1 , while in the weak measurements $(d)-(f)$ the peak of the distribution is located in the weak value $\left(\sigma_{\xi}\right)_{w}=\left\langle\uparrow_{y}\left|\sigma_{\xi}\right| \uparrow_{x}\right\rangle /\left\langle\uparrow_{y} \mid \uparrow_{x}\right\rangle=\sqrt{2}$. The outcomes of the weak measurement on the ensemble of 5000 pre- and post-selected particles, $(f)$, are clearly outside the range of the eigenvalues, $(-1,1)$. 
This figure "fig1-1.png" is available in "png" format from: http://arxiv.org/ps/hep-th/9411196v2 

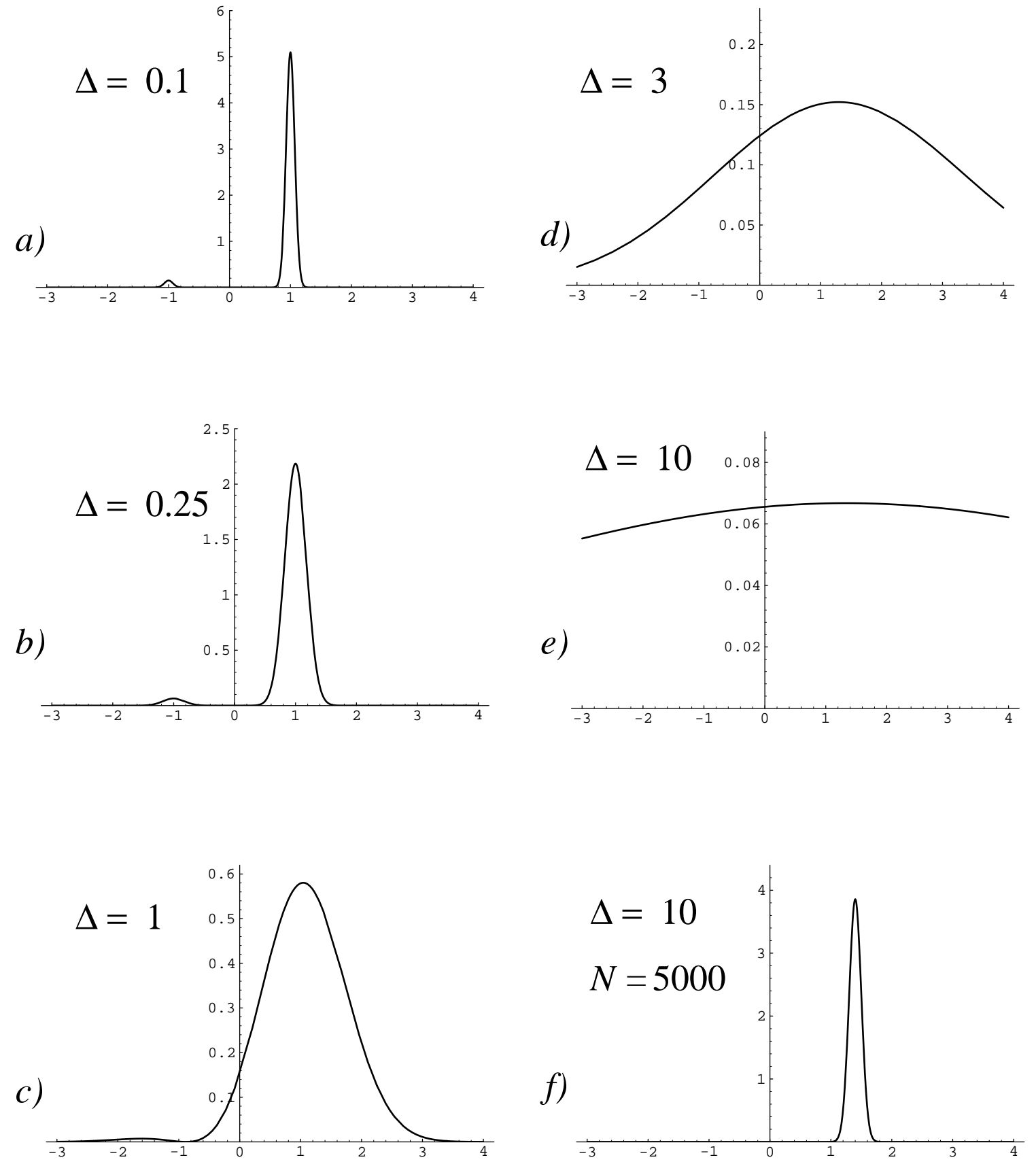